\title{
Design and experimental characterization of a tunable vibration-based electromagnetic micro-generator
}

\author{
Dibin Zhu ${ }^{\mathrm{a}, *}$, Stephen Roberts ${ }^{\mathrm{b}}$, Michael J. Tudor ${ }^{\mathrm{a}}$, Stephen P. Beeby ${ }^{\mathrm{a}}$ \\ a School of Electronics and Computer Science, University of Southampton, Southampton SO17 1BJ, UK \\ b Perpetuum Ltd, Epsilon House, Southampton Science Park, Southampton SO16 7NS, UK
}

\section{A R T I C L E I N F O}

\section{Article history:}

Received 29 June 2009

Received in revised form 8 December 2009

Accepted 4 January 2010

Available online 13 January 2010

\section{Keywords:}

Tunable

Micro-generator

Electromagnetic

Vibration energy harvesting

\begin{abstract}
A B S T R A C T
Vibration-based micro-generators, as an alternative source of energy, have become increasingly significant in the last decade. This paper presents a new tunable electromagnetic vibration-based micro-generator. Frequency tuning is realized by applying an axial tensile force to the micro-generator. The dimensions of the generator, especially the dimensions of the coil and the air gap between magnets, have been optimized to maximize the output voltage and power of the micro-generator. The resonant frequency has been successfully tuned from 67.6 to $98 \mathrm{~Hz}$ when various axial tensile forces were applied to the structure. The generator produced a power of 61.6-156.6 $\mu \mathrm{W}$ over the tuning range when excited at vibrations of $0.59 \mathrm{~m} \mathrm{~s}^{-2}$. The tuning mechanism has little effect on the total damping. When the tuning force applied on the generator becomes larger than the generator's inertial force, the total damping increases resulting in reduced output power. The resonant frequency increases less than indicated from simulation and approaches that of a straight tensioned cable when the force associated with the tension in the beam becomes much greater than the beam stiffness. The test results agree with the theoretical analysis presented.
\end{abstract}

(C) 2010 Elsevier B.V. All rights reserved.

\section{Introduction}

The last decade has seen an increasing interest in the development of wireless sensor networks (WSN). Wireless systems offer several advantages over a wired system, for example, they are flexible, easy to deploy and they can be placed in previously inaccessible locations. Furthermore, the layout of nodes in the wireless system can be easily changed without considering cabling. WSN can be widely used in monitoring of the environment, machine and structural health, surveillance, military, health, and security.

Since wireless sensors have no physical connection to the outside world, they must have their own power supply. At present, the conventional power source for wireless sensor networks is a battery. However, a battery has some disadvantages: it is quite large, can supply only a finite amount of energy and contains chemicals be hazardous. For some applications, WSN are deployed in harsh environments which may be difficult to access to replace the batteries. Therefore, it is preferred to make the sensors self-contained with their own renewable power supply. Recent advances in lowpower sensor technology have reduced power requirements to the

\footnotetext{
* Corresponding author. Tel.: +44 238059 5161; fax: +44 2380592901.

E-mail addresses: dz@ecs.soton.ac.uk(D.Zhu), stephen.roberts@perpetuum.co.uk(S. Roberts), mjt@ecs.soton.ac.uk (M.J. Tudor), spb@ecs.soton.ac.uk (S.P. Beeby).
}

level of only several milliwatts [1,2], which makes the concept of a self-powered WSN feasible.

Some possible energy sources for WSN include photonic energy [3], thermal energy [4] and mechanical energy [5]. These sources can be used to replace or recharge the battery and increase the lifetime and capacity of WSN. Among these sources, photonic energy has already been widely used in power supplies. Solar cells provide excellent power density. However, energy harvesting using light sources restricts the working environment of sensors. Such sensors cannot normally work in low light or dirty conditions. Thermal energy can be converted to electrical energy by the Seebeck effect but the working environment for thermo-powered sensors is limited. Mechanical energy can be found in instances where thermal or photonic energy is not suitable, which makes extracting energy from mechanical energy an attractive alternative approach for powering wireless sensors. The source of mechanical energy can be the moving human body or a vibrating structure. The frequency of the mechanical excitation depends on the source: less than $10 \mathrm{~Hz}$ for human movements and typically over $30 \mathrm{~Hz}$ for machinery vibrations [6]. The research covered in this paper is based on a vibration-based micro-generator.

The majority of generators designed for vibration energy harvesting are based upon a spring-mass system which produces maximum power when its resonant frequency matches the ambient vibration frequency [7]. Furthermore, devices are typically designed to have a high $\mathrm{Q}$-factor to generate maximum power 
from low amplitude vibrations. However, in such cases, the output power drops significantly if the predominant ambient frequency and the device resonant frequency do not match. Most reported generators are designed to work only at one fixed resonant frequency [5]. This drawback severely limits the practical application of vibration-based micro-generators.

To date, there are generally two possible solutions to this problem. The first is to widen the bandwidth of the generator. However, there is a tradeoff between the system bandwidth and the Q-factor. Wider bandwidth means a lower Q-factor, which reduces the maximum power generated. This can be compensated by making a generator larger in size but this is not always a practical solution. Bandwidth can also be widened by designing a generator consisting of an array of small generators, each of which works at a different frequency. Thus, the assembled generator has a wide operational frequency range while the Q-factor does not decrease $[8,9]$. However, this assembled generator must be well designed so that each individual generator does not affect the others. This makes it more complex to design and fabricate. Additionally, at a particular source frequency, only a single individual generator contributes to power output so the approach is volume inefficient. Other methods of widening the bandwidth include using non-linear or bi-stable devices for energy harvesting, which are theoretically more complicated than linear generators. The second solution is to tune the resonant frequency of a single generator periodically so that it matches the frequency of ambient vibration at all times. Thus, the maximum power can be generated at various frequencies without reducing the Q-factor and with high efficiency per unit volume. Therefore, this second approach is a better choice to increase the working frequency range of a vibration-based micro-generator.

Attempts to tune the frequency of generators have been reported in the literature. Challa et al. [10] reported a tunable piezoelectric micro-generator, $50 \mathrm{~cm}^{3}$ in volume, with a frequency range of $22-32 \mathrm{~Hz}$ with a tuning distance of $3 \mathrm{~cm}$. Tuning distance is defined as the maximum distance that the tuning mechanism moves over the entire tuning range. Tuning was realized by manually applying a magnetic force perpendicular to the cantilever of the generator. The generator produced $240-280 \mu \mathrm{W}$ powers at $0.8 \mathrm{~m} \mathrm{~s}^{-2}$ acceleration but the tuning mechanism had the unwanted side effect of varying damping over the frequency range. Leland and Wright [11] successfully tuned the resonant frequency of a vibration-based piezoelectric generator by manually applying an axial compressive force directly on the cantilever using a micrometer. The tuning range was from 200 to $250 \mathrm{~Hz}$. This device generated $300-400 \mu \mathrm{W}$ at an acceleration of $9.8 \mathrm{~m} \mathrm{~s}^{-2}$ and the total damping of the generator increased significantly when the compressive force was applied.

In this paper, a novel tunable vibration-based electromagnetic micro-generator is reported. Theoretical analysis of inertial generators, the principle of frequency tuning, design methods and experimental results of the generator are presented. This tunable micro-generator has a tuning range from 67.6 to $98 \mathrm{~Hz}$. It produced a power of $61.6-156.6 \mu \mathrm{W}$ over the tuning range when excited at vibrations of $0.59 \mathrm{~m} \mathrm{~s}^{-2}$. Additionally, the total damping of the generator maintains constant over $60 \%(67.6-85 \mathrm{~Hz})$ of the entire tuning range.

\section{Basic theory}

\subsection{Vibration-based micro-generator}

Vibration-based micro-generators can be modelled using second-order, spring-mass systems [7]. The average power dissipated within the damper (both by transduction mechanism and parasitic damping mechanisms) is given by [7]:

$$
P(\omega)=\frac{m \zeta_{T} Y^{2}\left(\omega / \omega_{r}\right)^{3} \omega^{3}}{\left[1-\left(\omega / \omega_{r}\right)^{2}\right]^{2}+\left[2 \zeta_{T}\left(\omega / \omega_{r}\right)\right]^{2}}
$$

where $m$ is the mass, $\zeta_{T}$ total damping factor, $Y$ is the amplitude of vibration, $\omega_{r}$ is the resonant frequency and $\omega$ is the angular frequency of vibration.

The maximum power available within the vibration-based micro-generator, $P_{\max }$, occurs when the resonant frequency equals the vibration frequency, i.e. $\omega=\omega_{r}$, and is given by [5]:

$P_{\max }=\frac{m \cdot a_{v}^{2}}{4 \cdot \omega_{r} \cdot \zeta_{T}}$

where $a_{v}$ is the acceleration level and $a_{v}=Y \omega^{2}$.

The total damping factor is the sum of the electrical damping factor, $\zeta_{e}$ and the mechanical damping factor, $\zeta_{m}$. For maximum efficiency, the power transferred to the electrical load should equal the mechanical losses, i.e. $\zeta_{e}=\zeta_{m}$. Therefore, the maximum power delivered to the electrical domain is given by:

$P_{E_{-} \max }=P_{\max } \cdot \frac{\zeta_{e}}{\zeta_{m}+\zeta_{e}}=\frac{m \cdot a_{v}^{2}}{16 \cdot \omega_{r} \cdot \zeta_{m}}$

For an electromagnetic generator which has a coil with a resistance $R_{\text {coil }}$, the power transferred into a resistive load $\left(R_{\text {load }}\right), P_{L}$ is given by:

$P_{L}=P_{E_{-} \max } \cdot\left(\frac{R_{\text {load }}}{R_{\text {load }}+R_{\text {coil }}}\right)=\frac{m \cdot a_{v}^{2}}{16 \cdot \omega_{r} \cdot \zeta_{m}} \cdot\left(\frac{R_{\text {load }}}{R_{\text {load }}+R_{\text {coil }}}\right)$

Eq. (4) shows that the maximum power generated by the microgenerator decreases when the resonant frequency increases for constant acceleration and constant damping.

\subsection{Frequency tuning}

Many generator designs employ a cantilever spring element with an inertial mass at the free end [5]. An axial tensile load applied to a cantilever increases the resonant frequency of the cantilever while an axial compressive load decreases the resonant frequency.

An approximate formula for the resonant frequency of a uniform cantilever in mode $i$ with an axial load, $f_{r i}^{\prime}$, is given by [12]:

$f_{r i}^{\prime}=f_{r i} \cdot \sqrt{1+\frac{F}{F_{b}} \cdot \frac{\lambda_{1}^{2}}{\lambda_{i}^{2}}}$

where $f_{r}$ is the resonant frequency in mode $i$ without load and $F$ is the axial load. $F$ is positive if the load is tensile and $F$ is negative if the load is compressive. $F_{b}$ is the compressive axial load required to buckle the beam. $\lambda_{1}$ is a dimensionless load parameter which is a function of and the beam boundary conditions applied to the cantilever for mode 1 of the beam and $\lambda_{i}$ is the same parameter for mode $i . \lambda_{i}$ is given by the $i$ th solution of Eq. (6) [12].

$\cos \lambda \cdot \cosh \lambda+1=0$

Since most micro-generators with a beam structure work at a resonance of mode 1 , the resonant frequency of a uniform cantilever in mode 1 with an axial load, $f_{r 1}^{\prime}$, is given by:

$f_{r 1}^{\prime}=f_{r 1} \cdot \sqrt{1+\frac{F}{F_{b}}}$

The buckling load of a cantilever, $F_{b}$, is given by [12]:

$F_{b}=\frac{\pi^{2} \cdot E \cdot I}{4 L^{2}}$

where $E$ is the Young's modulus of the material of the cantilever, $I$ is the area moment of inertia and $L$ is the length of the cantilever. 


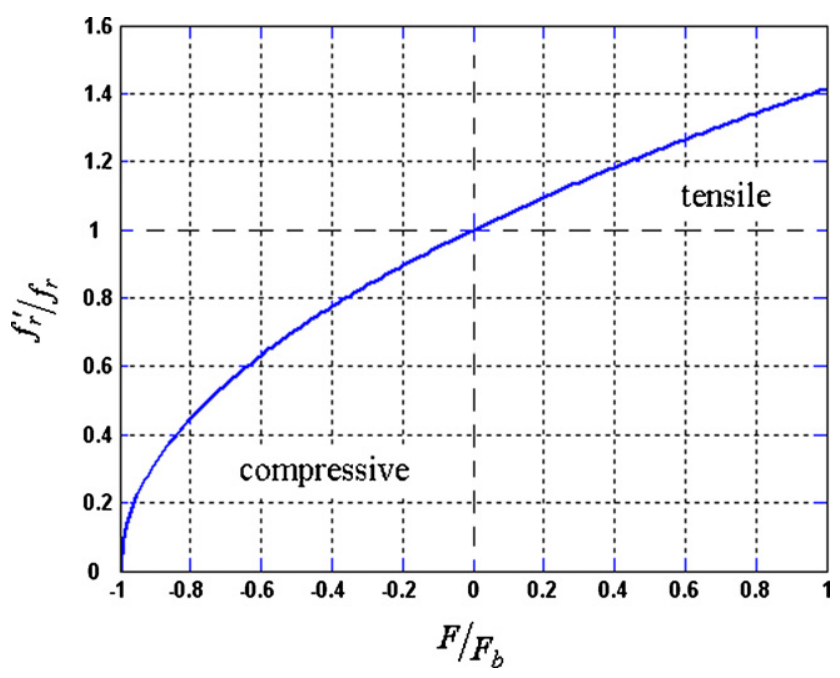

Fig. 1. Change in resonant frequency with axial load.

Fig. 1 shows the change in the resonant frequency of a cantilever with a varying axial load. It shows that a compressive load is more efficient at frequency tuning than a tensile load. However, a preliminary test [13] showed that the parasitic damping of the generator rises with increasing axial compressive load. When an axial tensile load is applied, the damping levels remain constant over most of the tuning range. The total damping only increases when the tensile force becomes large compared to the excitation force. Therefore, a tensile load is preferred in this application. The effect of the tensile force on the total damping of the cantilever will be discussed in Section 2.4. To minimize unwanted effects on the cantilever vibrations, contactless magnetic force provided by two tuning magnets is used to apply the axial load. Fig. 2 shows the schematic diagram of the frequency tuning mechanism.

When the tensile load applied to the cantilever becomes much greater than the buckling force, the resonant frequency of the cantilever approaches that of a straight tensioned cable with an off-centre mass because the force associated with the tension in the beam becomes much greater than the beam stiffness. This frequency limit is given by [12]:

$f_{r}=\frac{1}{2 \pi} \cdot \sqrt{\frac{3 E \cdot I \cdot\left(l_{1}+l_{2}\right)}{m \cdot l_{1}^{2} \cdot l_{2}^{2}}}$

where $l_{1}$ and $l_{2}$ are distances from the centre of gravity of the mass to both fixed ends.

\subsection{Calculation of magnetic tuning force}

The calculation of magnetic force between simple shape magnets can be performed numerically. Only the key equations are

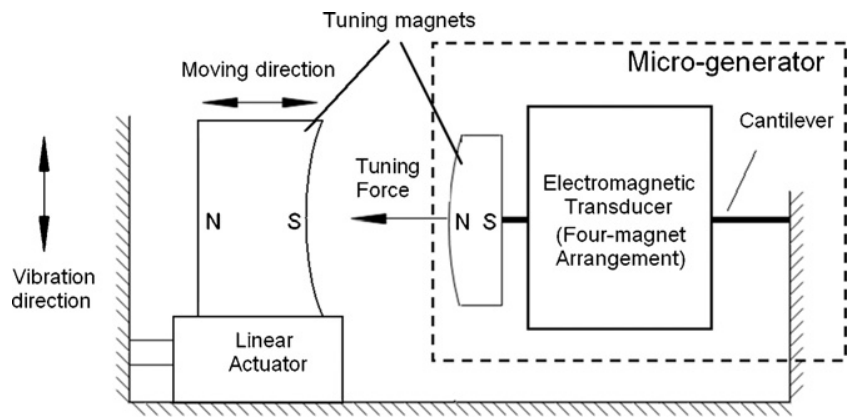

Fig. 2. Schematic diagram of tuning mechanism.

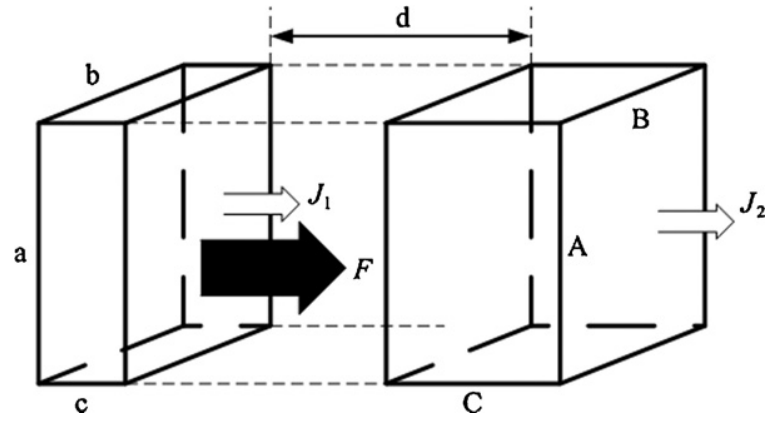

Fig. 3. Magnet configuration.

given here. A detailed description of this method can be found in [14]. For two cuboid magnets sharing the same central line along their thickness and with the area where these two magnets face each other, perfectly overlapping, as shown in Fig. 3, the magnetic attractive force between them can be calculated using:

$F=\frac{J_{1} \cdot J_{2}}{4 \pi \mu_{0}} \sum_{i=0}^{1} \sum_{j=0}^{1} \sum_{k=0}^{1} \sum_{l=0}^{1} \sum_{p=0}^{1} \sum_{q=0}^{1}(-1)^{i+j+k+l+p+q} \phi\left(u_{i j}, v_{k l}, w_{p q}, r\right)$

where $J_{1}$ and $J_{2}$ are the magnetization of these two magnets, respectively. $\mu_{0}$ is the magnetic constant and $\phi\left(u_{i j}, v_{k l}, w_{p q}, r\right)$ is a function of dimensions of the two magnets and their relative position. For the magnet configuration shown in Fig. 3 to be analyzed here, the interactive force between the two magnets is parallel with their polarization. Therefore, $\phi$ is given by:

$$
\begin{aligned}
\phi\left(u_{i j}, v_{k l}, w_{p q}, r\right)= & -u_{i j} \cdot w_{p q} \cdot \ln \left(r-u_{i j}\right)-v_{k l} \cdot w_{p q} \cdot \ln \left(r-v_{k l}\right) \\
& +u_{i j} \cdot v_{k l} \cdot \tan ^{-1} \frac{u_{i j} \cdot v_{k l}}{r \cdot w_{p q}}-r \cdot w_{p q}
\end{aligned}
$$

where $u_{i j}=(-1)^{j} \cdot A-(-1)^{i} \cdot a$

$$
\begin{aligned}
& v_{k l}=(-1)^{l} \cdot B-(-1)^{k} \cdot b \\
& w_{p q}=d+\frac{C+c}{2}+(-1)^{q} \cdot C-(-1)^{p} \cdot c \\
& r=\sqrt{u_{i j}^{2}+v_{k l}^{2}+w_{p q}^{2}} .
\end{aligned}
$$

In this particular application, the areas where the two magnets face each other are curved to maintain a constant gap between them over the amplitude range of the generator so that the axial tuning force remains constant when the distance between the two tuning magnets is fixed. Since the curvatures are small, as an approximation, they can be regarded as two rectangular parallelepipedic magnets so that Eq. (10) can be applied. Fig. 4 shows the theoretical tuning force for this particular case based on Eqs. (10) and (11). The designated minimum and maximum distance between the two tuning magnets are 1.2 and $5 \mathrm{~mm}$, respectively to give the generator a tuning range of $30 \mathrm{~Hz}$. The estimated tuning forces are from 1 to $6.67 \mathrm{~N}$.

\subsection{Q-factor under tuning force}

In this section, the effect of the tuning force on the Q-factor of the generator is studied. Fig. 5 shows the forces on the resonator along the $z$ axis. To make this figure easy to understand, the transducer is omitted as the tuning force is only applied on the tuning magnet 1 and it is assumed that the tuning magnet 1 represents the overall mass of the generator. The magnetic force along the $x$ and $y$ axes remains constant once the distance between the two magnets is fixed. In addition, the resonator is assumed to travel only along the 


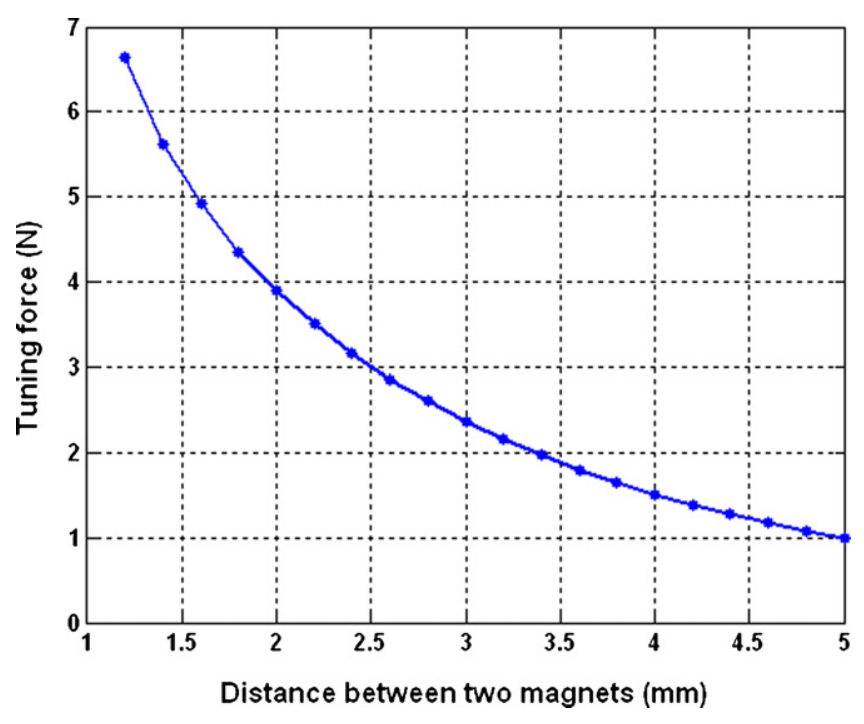

Fig. 4. Tuning force with variations of distances between two tuning magnets.

$z$ axis to simplify the analysis. As the resonator does not move along the $x$ and $y$ axes, the magnetic force along these two axes can be omitted. Therefore, only forces along the $z$ axis have to be studied.

The dynamic model in this situation is given by:

$m \frac{d^{2} z(t)}{d t^{2}}+b \frac{d z(t)}{d t}+k z(t)+F_{T_{-}}[z(t)]=F_{i}(t)$

where $m$ is the mass, $b$ is the damping coefficient, $k$ is the spring constant and $z(t) F_{T_{\mathcal{Z}}}[z(t)]$ is the $z$ component of the magnetic force introduced by the two tuning magnets, which can be calculated using Eq. (10). The interactive force between the two magnets is perpendicular to their polarization. Thus, in this case, the function $\phi$ in Eq. (10) is given by:

$$
\begin{aligned}
\phi\left(u_{i j}, v_{k l}, w_{p q}, r\right)= & \frac{1}{2}\left(u_{i j}^{2}-w_{p q}^{2}\right) \cdot \ln \left(r-v_{k l}\right)+u_{i j} \cdot v_{k l} \cdot \ln \left(r-u_{i j}\right) \\
& +u_{i j} \cdot w_{p q} \cdot \tan ^{-1} \frac{u_{i j} \cdot v_{k l}}{r \cdot w_{p q}}+\frac{1}{2} r \cdot v_{k l}
\end{aligned}
$$

$F_{i}(t)$ is the inertial force on the mass, which is given by:

$F_{i}(t)=-m \frac{d^{2} z_{h}(t)}{d t^{2}}$

where $z_{h}(t)$ is the displacement of the vibration source.

The $\mathrm{Q}$-factor of the generator is given by:

$Q=\frac{Z}{Z_{h}}$

where $Z$ and $Z_{h}$ are the maximum values of $z(t)$ and $z_{h}(t)$, respectively.

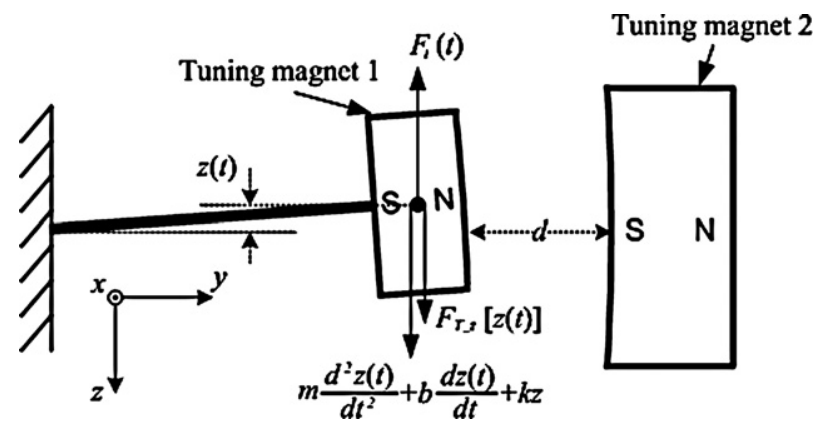

Fig. 5. Forces on the resonator.
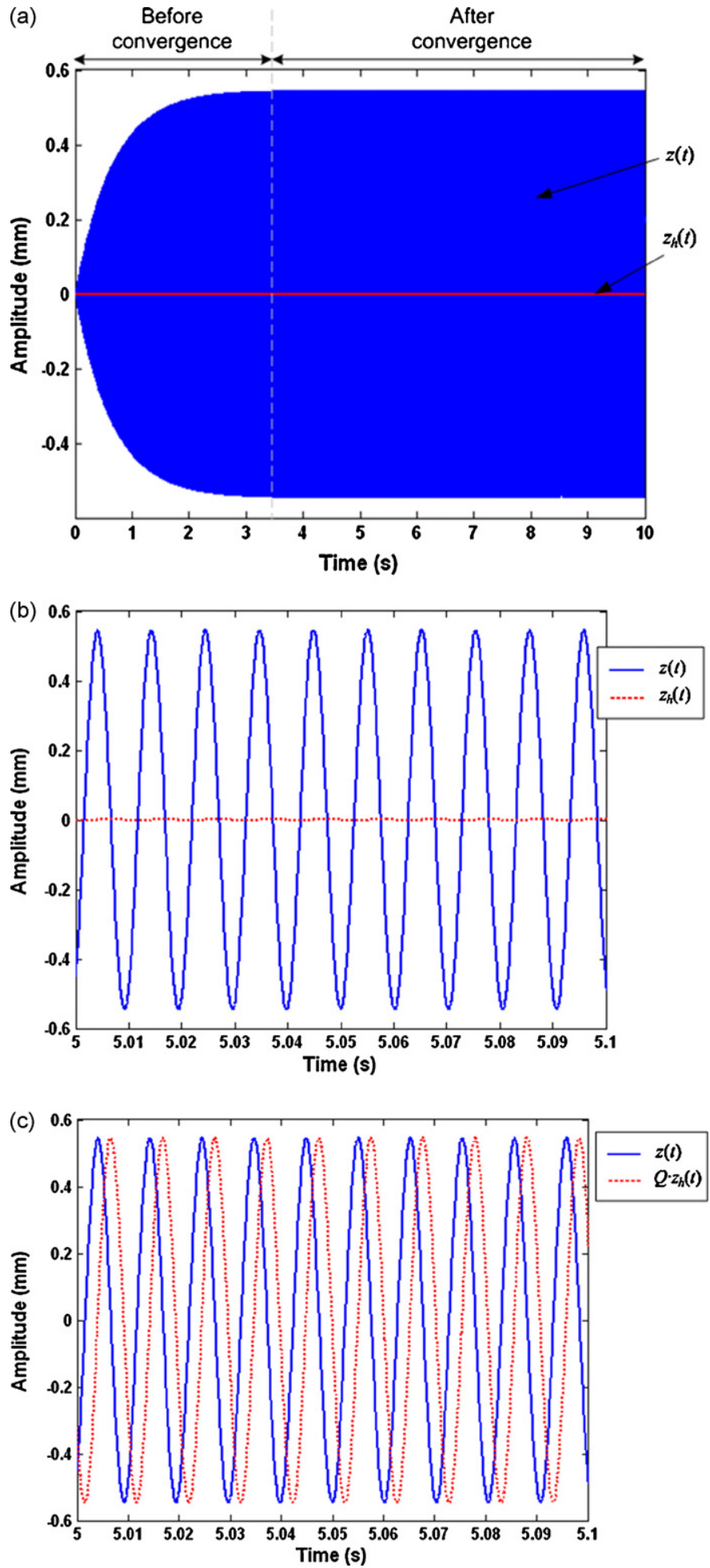

Fig. 6. Numerical solution of differential Eq. (14) (a) overall waveform, (b) detailed waveform (comparison of amplitude), and (c) detailed waveform (comparison of phase).

As it is difficult to solve analytically the differential Eq. (12), numerical methods are used to obtain a solution. In this case, a fourth-order Runge-Kutta method was adopted [15]. Fig. 6 shows an example of the numerical solution to Eq. (14). The waveform after the solution converged was treated as an approximation to the actual solution as shown in Fig. 6(a). In Fig. 6(b) and (c), detailed waveforms (after the solution converged) of $z(t)$ and $z_{h}(t)$ are given to compare their respective amplitudes and phases. The Q-factor 


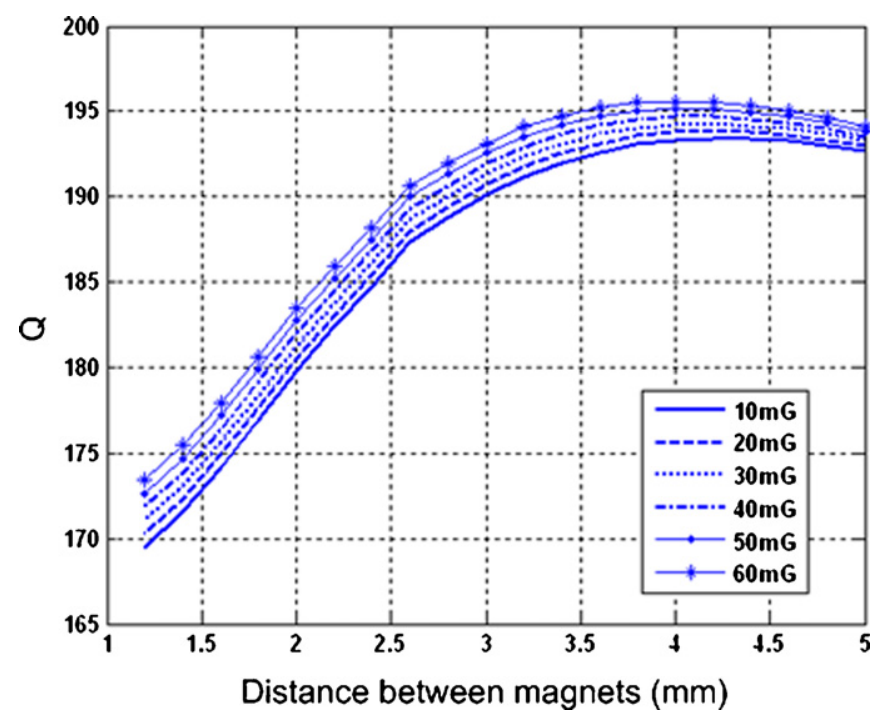

Fig. 7. Q-factor with various distances between two tuning magnets and acceleration levels.

can be obtained by observing the maximum value of $z(t)$ and $z_{h}(t)$ in Fig. 6(b).

Fig. 7 shows the calculated Q-factor with various distances between the two tuning magnets and at various acceleration levels. It was found that, as the excitation level increases, the damping effect from the tuning magnets decreases. This enables the tuning magnets to be brought closer together at higher excitation levels without reducing the $\mathrm{Q}$-factor and therefore a wider tuning range can be obtained.

\section{Tunable vibration-based electromagnetic micro-generator design}

The electromagnetic micro-generator presented in this paper is a modified version of a design previously developed as part of an EU funded research project 'VIBES' [16]. Fig. 8 shows the overall design of the tunable vibration-based electromagnetic micro-generator. In Fig. 9(c), a DIP-16 socket is placed next to the generator as a reference of its dimensions.

\subsection{Electromagnetic transducer}

The generator has a four-magnet structure as the generator designed in the 'VIBES' project [16]. Two mild steel keepers were used to couple the magnetic flux between the top and bottom magnets, which ensured a uniform magnetic field within the air gap. The coil was attached to the housing of the generator. The fourmagnet structure was fixed to a cantilever beam and vibrated with the ambient vibration. The magnets moved with respect to the static coil so that an induced current was generated within the coil according to Faraday's law.

\subsection{Tuning mechanism}

Recall the schematic diagram of the tuning mechanism as shown in Fig. 2; the generator and the tuning mechanism were mounted on the same housing so that there was no relative displacement between them. The tuning force was provided by the attractive force between the two tuning magnets with opposite poles facing each other. One magnet was fixed to the free end of a cantilever while the other was attached to an actuator and placed axially in line with the cantilever. The tuning magnet attached to the actuator was larger than the one on the generator along the vibration
Table 1

Material of each component.

\begin{tabular}{lll}
\hline Component & Material & Reason for selection \\
\hline Magnet & NdFeB & High energy density \\
Keeper & Mild steel & Ferromagnetic material \\
Additional mass & Tungsten alloy & High density \\
Beam & BeCu & Excellent fatigue characteristics \\
Base & Tecatron GF40 & High rigidity, non-ferromagnetic \\
\hline
\end{tabular}

direction so that they always overlapped perfectly during operation. The distance between the two tuning magnets was adjusted by the linear actuator. Thus the axial load on the cantilever was changed.

\subsection{Micro-generator design}

Each component of the generator was fabricated separately using conventional manufacturing processes. The magnets, the mild steel keeper and the additional tungsten mass were glued to the cantilever beam with cyanoacrylate with the aid of an alignment jig. This assembly was then clamped onto the base using an M1 sized nut and a copper washer. The coil was bonded to a pre-machined coil support on the base. Table 1 summarizes the materials used for each component and the reasons for their selection.

\subsubsection{Cantilever beam}

The resonant frequency of the generator is controlled by the dimensions of the beam and the inertial mass. For a given inertial mass of $2.4 \mathrm{~g}$, a beam length of $13 \mathrm{~mm}$ and a width of $5 \mathrm{~mm}$, the untuned resonant frequency and tuning range when the tuning force changes from 1 to $6.67 \mathrm{~N}$ as a function of beam thicknesses are shown in Fig. 9. These results were obtained from a prestressed ANSYS modal analysis. It was found that the thinner the beam, i.e. the lower the spring constant of the cantilever, the lower the untuned resonant frequency and the larger the tuning range. For this generator, a $120 \mu \mathrm{m}$ thick beam was chosen to give a predicted untuned resonant frequency of $45.2 \mathrm{~Hz}$ and a tuning range from 66.4 to $108.8 \mathrm{~Hz}$.

\subsubsection{Coil and air gap}

According to Faraday's law, the induced voltage within the coil is given by:

$V=-N \frac{d \Phi}{d t}$

where $N$ is the number of turns of the coil and $d \Phi / d t$ is the magnetic flux gradient through the coil. The number of turns of the coil is given by:

$N=\frac{4 F_{C} \cdot\left(R_{o}-R_{i}\right) \cdot t}{\pi \cdot d^{2}}$

where $F_{c}$ is the coil fill factor (the ratio of the volume of conductor to the volume of the coil), $R_{o}$ and $R_{i}$ are the outer and inner radius of the coil, respectively. $t$ is the thickness of the coil and $d$ is the diameter of the coil wire.

In order to maximize the induced voltage, the number of coil turns and the magnetic flux gradient should be maximized. The limitation of generator size and coil winding capability fix the outer and inner radii of the coil. The wire with a diameter of $16 \mu \mathrm{m}$ was chosen to give the coil the maximum number of turns compatible with winding and handling. The fill factor is a function of the winding process and is typically $0.5-0.6$. Therefore, the only parameter that can be changed to increase the number of turns is the coil thickness. Increasing coil thickness linearly increases the number of turns as shown in Fig. 10(a). However, making the coil thicker 
(a)

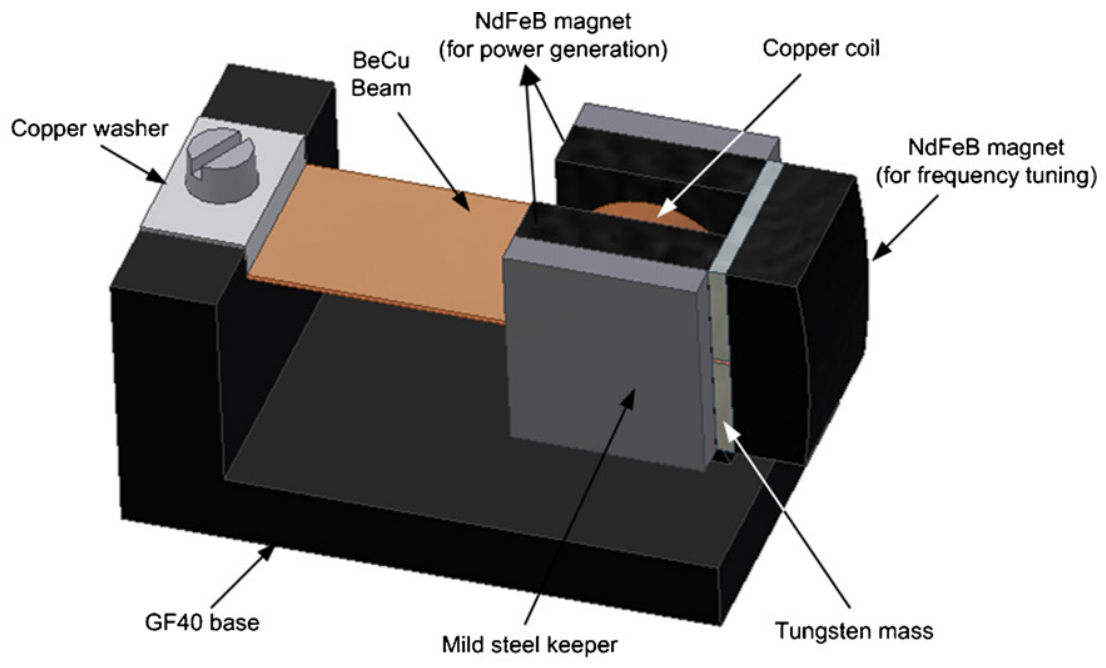

(b)

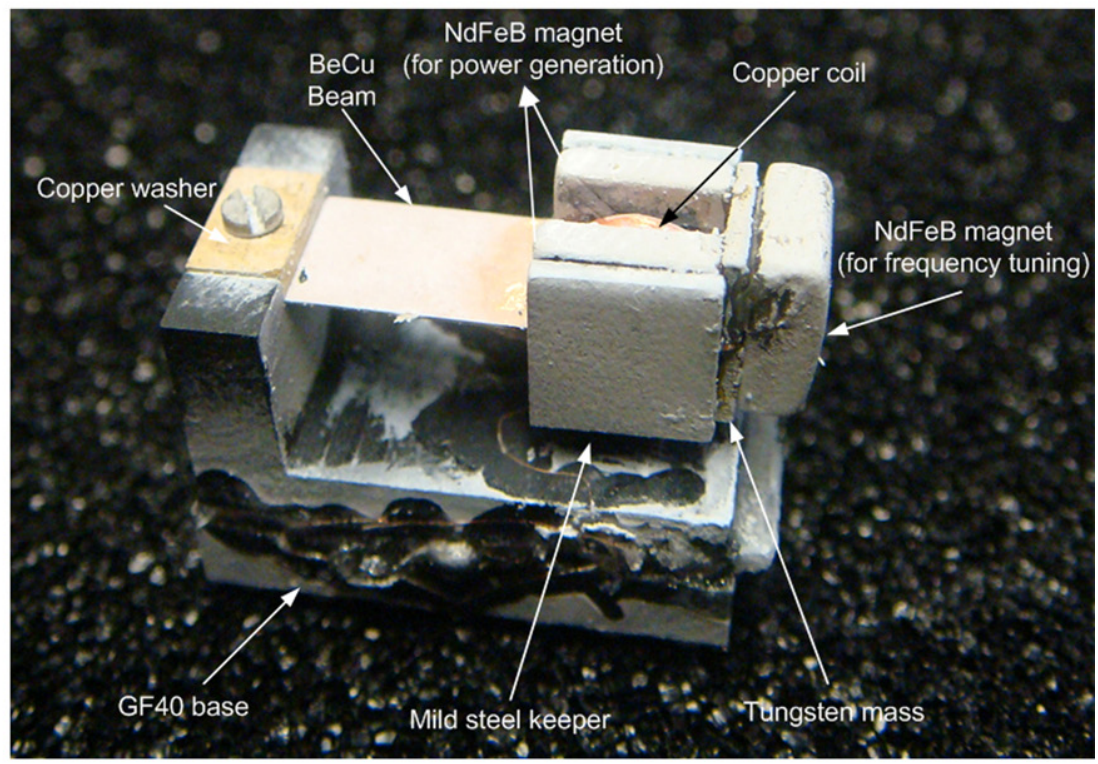

(c)

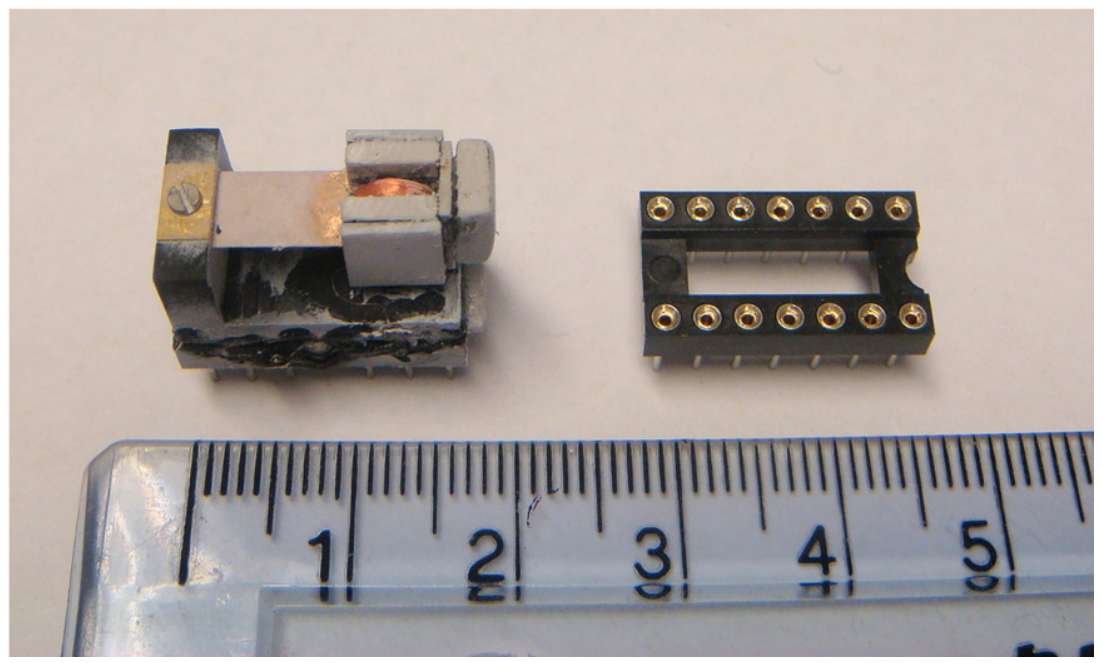

Fig. 8. Tunable electromagnetic micro-generator (a) model, (b) photo, and (c) generator with indication of its dimensions. 


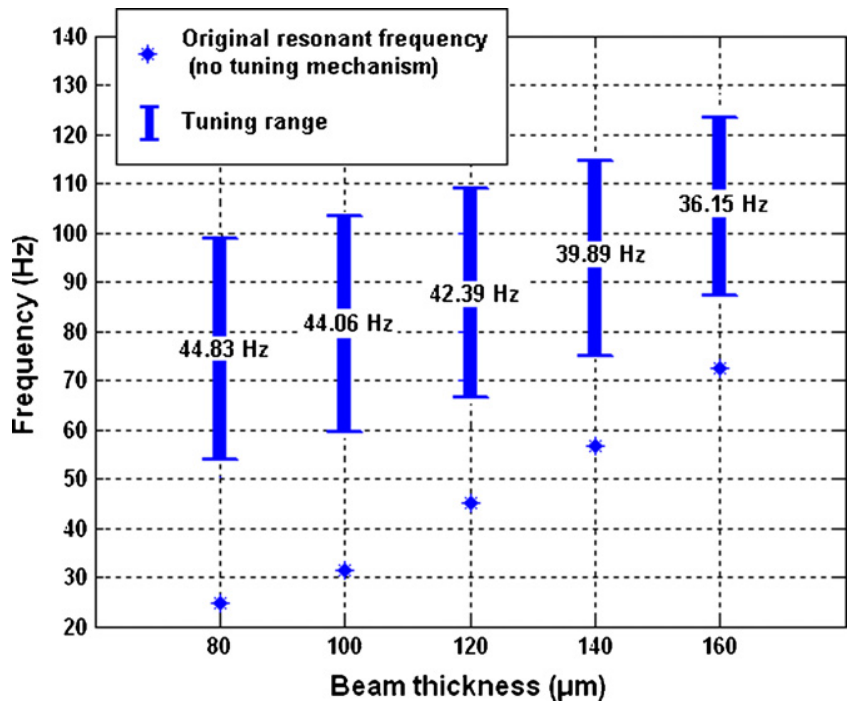

Fig. 9. Base resonant frequency and tuning range with variation of beam thicknesses.

means that the air gap between the magnets has to be increased to avoid impact between the magnets and the coil. Based on magnetic circuit theory, a larger air gap reduces the magnetic flux within the magnetic circuit as shown in Fig. 10(b). Hence, there is a tradeoff between the coil thickness and the magnetic flux through the coil. For a constant gap between coil and magnets of $0.3 \mathrm{~mm}$, simulation shows that the optimum air gap and coil thickness are 1.9 and $1.3 \mathrm{~mm}$, respectively. The estimated number of turns is 6950 when the fill factor is 0.5 and this gives a voltage output of $8.1 \mathrm{~V}$ as shown in Fig. 10(c).

\subsubsection{Magnetic field modelling}

As mentioned in Section 3.1, the tunable generator presented here has the same magnetic circuit as the generator designed in the 'VIBES' project [16]. The only difference is the existence of the tuning magnet at the free end of the cantilever. The effect of these tuning magnets on the magnetic circuit of the four-magnet arrangement was simulated using Ansoft Maxwell 3D magnetic finite element (FE) software. Fig. 11 shows the simulation results of the magnetic filed and compare the magnetic field of the generator with and without tuning magnets. It is found that the magnetic field within the air gap is barely affected by the tuning magnets. It can be seen from Fig. 11 that the maximum flux density within the air gap is $0.45 \mathrm{~T}$ with or without the tuning magnet in the simulation. The simulation results agree with the mathematical calculations discussed in Section 3.3.2 (Fig. 10(b)).

\section{Experimental results and analysis}

The generator was tested on a shaker table with a programmable resistance box and a PC with LabVIEW software collecting the data. This system is suitable for fully characterizing the generator over a wide range of acceleration levels, load resistances and frequencies. The test platform of the system is shown in Fig. 12. A tuning magnet (5) is attached to the free end of the cantilever resonator on the generator (4). The other tuning magnet (3) is fixed on a slider (2) which is able to slide along a track. The movement of the slider is controlled by the linear actuator (1). The linear actuator used here is a Haydon ${ }^{\circledR} 21000$ Series Size 8 linear stepper motor, $\mathrm{E} 21 \mathrm{H} 4(\mathrm{AC})-5$. The minimum step length is $0.05 \mathrm{~mm}$. The distance between the two tuning magnets can be precisely controlled by counting the number of steps the stepper motor has executed via a micro-controller.
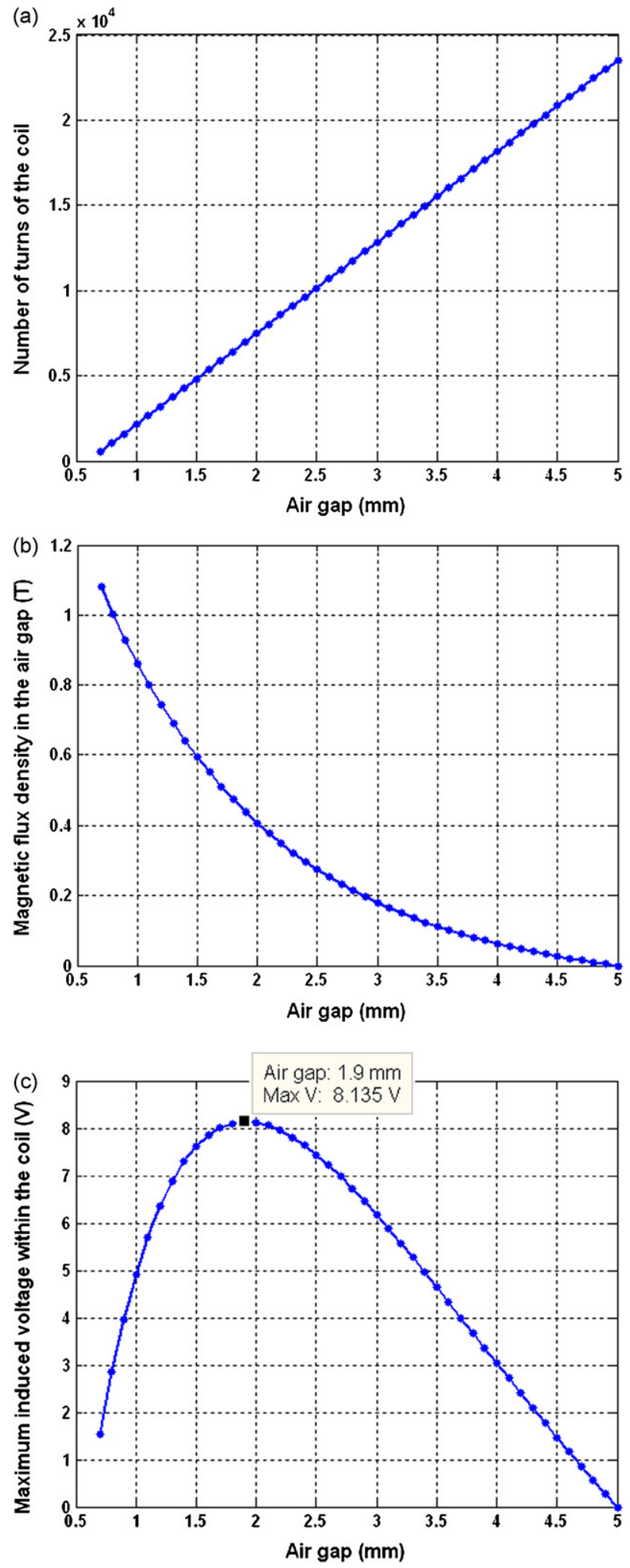

Fig. 10. Simulation results (a) coil turns with variation of air gaps, (b) magnetic flux density with variation of air gaps, and (c) maximum induced coil voltage with variation of air gaps.

\subsection{Resonant frequency}

The variation of the resonant frequency of the generator with the distance between the two tuning magnets is shown in Fig. 13(a). The resonant frequency increases as the distance between the two 
(a)

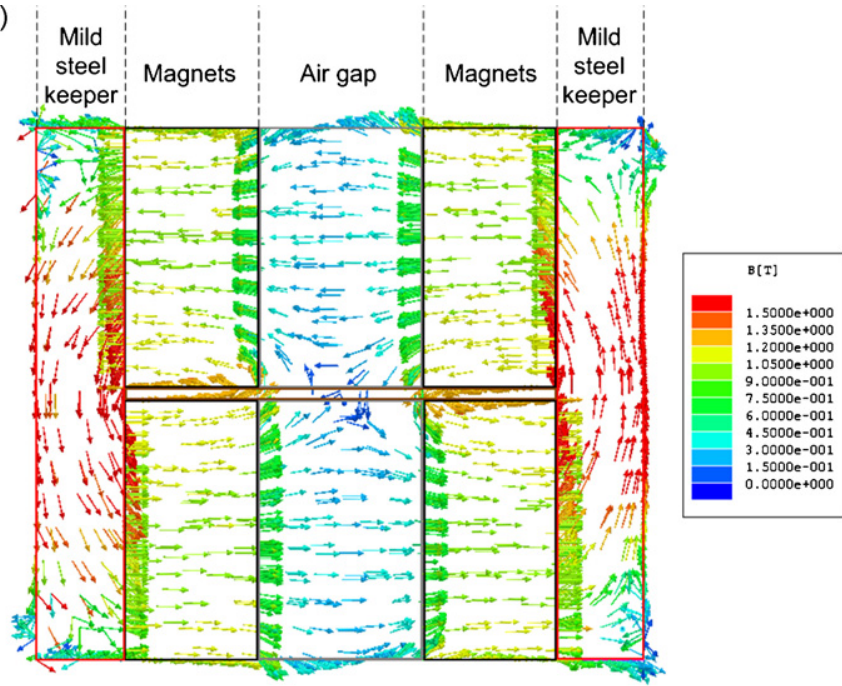

(b) i

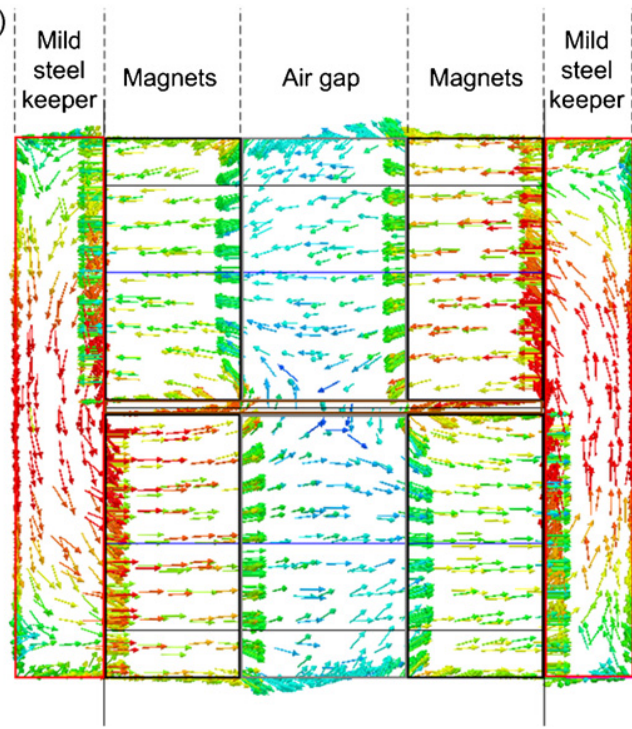

Fig. 11. Modelling of magnetic field (a) no tuning magnets and (b) with tuning magnets.

tuning magnets decreases, i.e. when the tuning force increases as in Fig. 13(b). The tuning range of the generator varies from 67.6 to $98 \mathrm{~Hz}$ when the distance between two tuning magnets changes from 5 to $1.2 \mathrm{~mm}$. The test results follow the simulation results until the distance between the two tuning magnets is less than $3 \mathrm{~mm}$. When this distance is less than $3 \mathrm{~mm}$, i.e. tuning force was larger than $2.87 \mathrm{~N}$, the resonant frequency increases less than simulated. The reason for this is as follows. As the force is time-varying as mentioned in Section 2.4, it is difficult to simulate. Therefore, only the axial force is taken into account in the simulation. When the two tuning magnets are relatively far away from each other, the force parallel to the vibration direction is negligible compared to the excitation force. So in this situation the test results agree with the simulation. However, when the two tuning magnets are closer, the parallel force starts to have more effect on the cantilever. As this was not simulated, the disagreement between the test results and simulation results becomes larger.

As mentioned in Section 2.2, when the tensile force is much greater than the buckling force of the cantilever, the resonant frequency will approach the resonant frequency of a straight tensioned cable with an off-centre mass. The buckling force of this cantilever is $0.5 \mathrm{~N}$ according to Eq. (8). The frequency limit of this

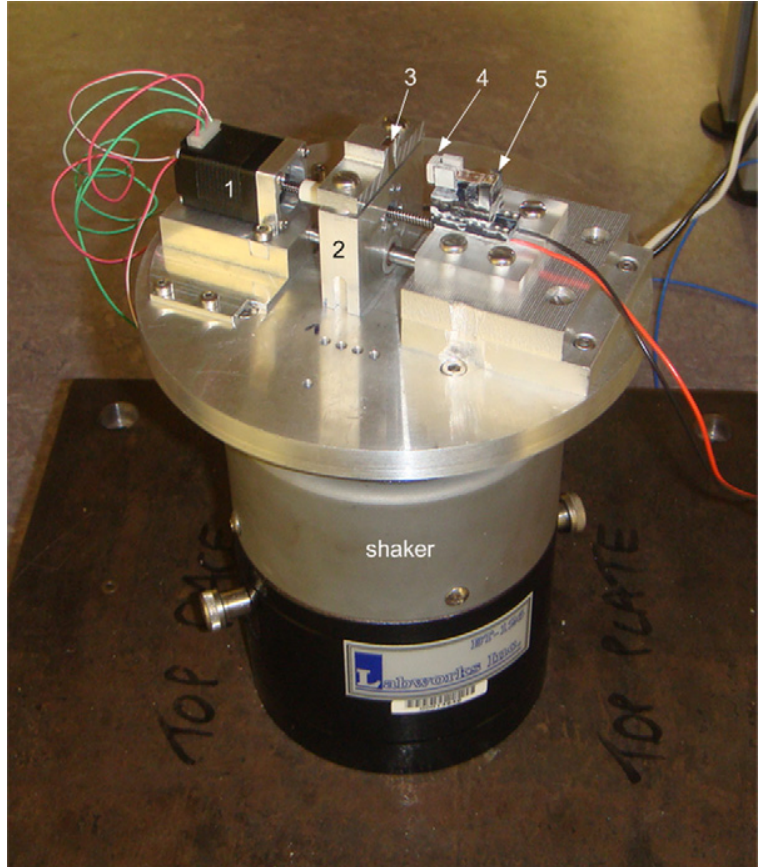

Fig. 12. Test setup of the mechanically tunable electromagnetic generator. ((1) Linear actuator; (2) slider; (3) tuning magnet 1; (4) tuning magnet 2; (5) microgenerator.)

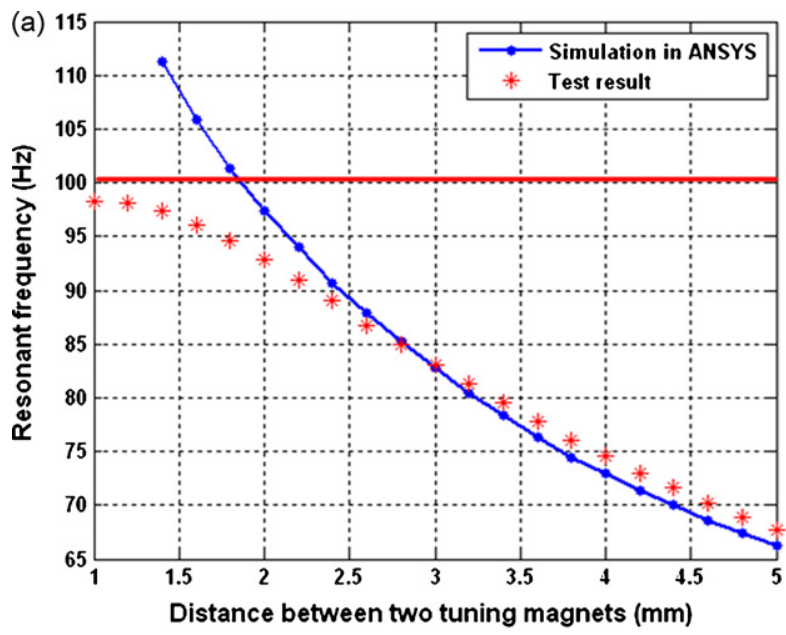

$100.3 \mathrm{~Hz}$

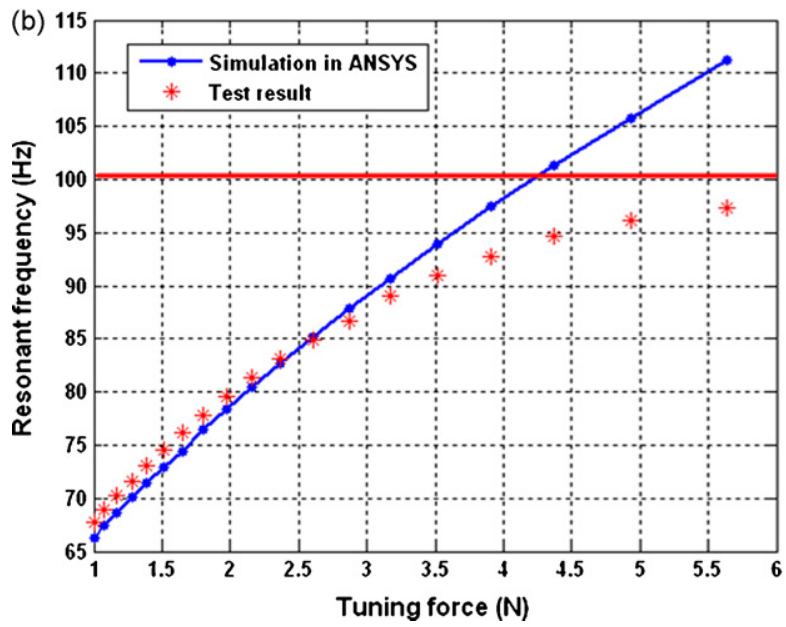

$100.3 \mathrm{~Hz}$

Fig. 13. Resonant frequency (a) with variation of distances between tuning magnets and (b) with variation of tuning force. 


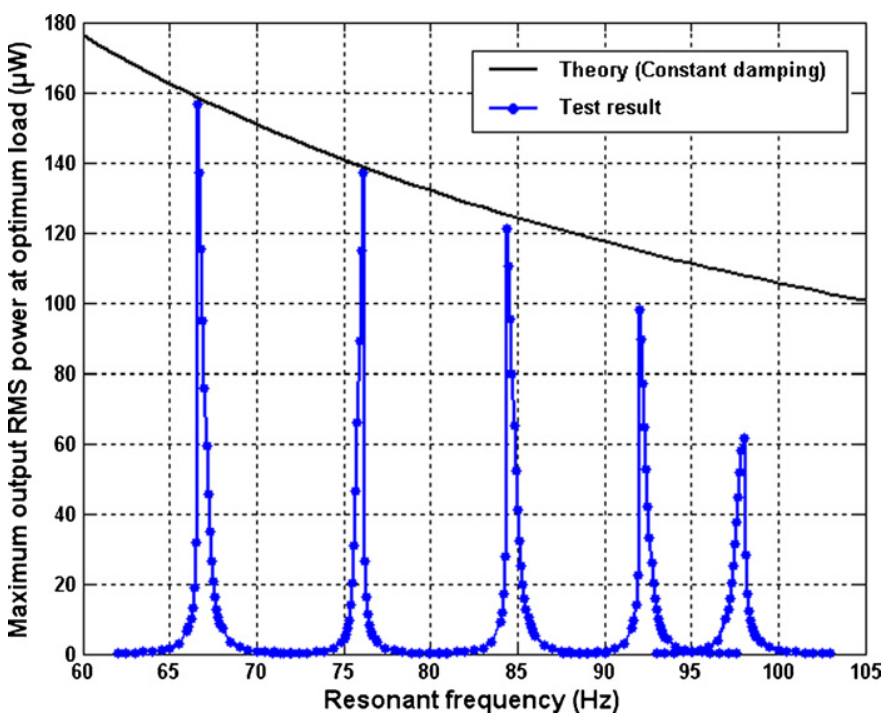

Fig. 14. Output RMS power at optimum loads with variation of tuned resonant frequencies of the generator (excited at $0.59 \mathrm{~m} \mathrm{~s}^{-2}$ ).

tunable generator is $100.3 \mathrm{~Hz}$ according to Eq. (9). The experimental results shown in Fig. 13 agree with the theoretical analysis presented in Section 2.2 .

\subsection{Generated power}

The maximum power at the optimum resistive load at different resonant frequencies when the micro-generator was excited at a vibration of $0.59 \mathrm{~m} \mathrm{~s}^{-2}$ is shown in Fig. 14. It was found that the maximum output power dropped with the increase of the resonant frequency as predicted from Eq. (4). However, when the two tuning magnets were less than $3 \mathrm{~mm}$ apart, i.e. when the resonant frequency was higher than $85 \mathrm{~Hz}$, the output power decreased more than expected. This result agreed with the test results of resonant frequency discussed in Section 4.1, which further proved that when the tuning force becomes too large, the total damping will increase and output power will reduce as predicted in Section 2.4.

Fig. 15 shows the maximum output RMS power at different excitation levels over the tuning range. It was found that the output power drop of more than that predicted by the constant damping model occurs at lower frequency if the excitation level was lower. In other words, the Q-factor drops at higher frequency if the exci-

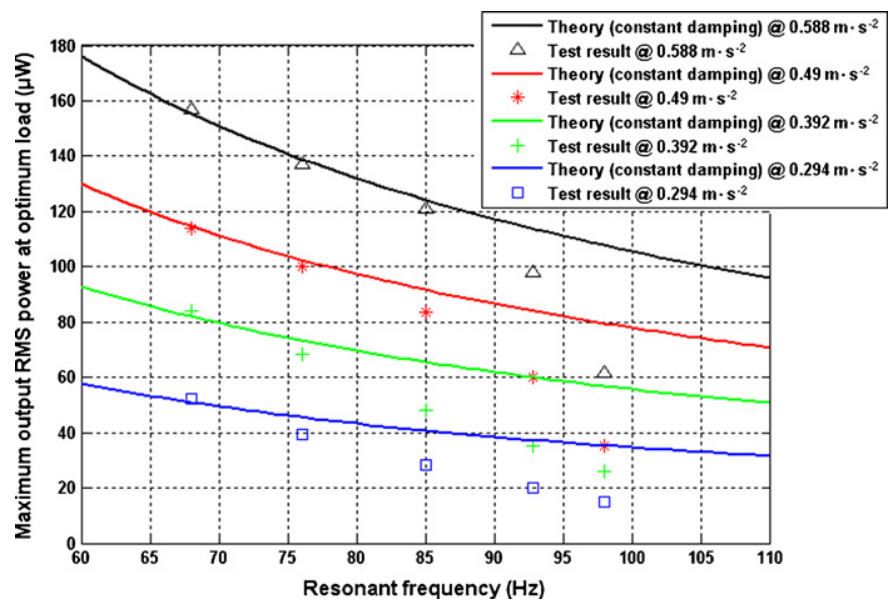

Fig. 15. Maximum output RMS power at different excitation levels. tation level is high, which agrees with the theoretical analysis in Section 2.4 .

\section{Conclusions}

This paper has presented a tunable vibration-based electromagnetic micro-generator. Its resonant frequency can be tuned by applying an axial tensile load using a pair of tuning magnets. The resonant frequency of the micro-generator can be tuned from 67.6 to $98 \mathrm{~Hz}$ by changing the distance between two tuning magnets from 5 to $1.2 \mathrm{~mm}$, respectively. The generator produced a power of 61.6-156.6 $\mu \mathrm{W}$ over the tuning range when excited at a constant low vibration acceleration level of $0.59 \mathrm{~m} \mathrm{~s}^{-2}$.

It is found that when the tensile force is much greater than the buckling force, the resonant frequency increases less than predicted from simulation and approaches a finite value. This is because the force associated with the tension in the beam becomes much greater than the beam stiffness and the resonant frequency approaches that of a straight tensioned cable.

Importantly, the devised tuning mechanism does not affect the damping of the micro-generator over $60 \%(67.6-85 \mathrm{~Hz})$ of the entire tuning range. The maximum output power drops with the increasing resonant frequency as predicted by the generator model with constant damping. However, when the tuning force becomes larger than the inertial force caused by vibration, total damping increases and the output power is less than that in the constant damping situation. In addition, an output power drop of more than the constant damping model indicates occurs at a lower force and hence, a lower frequency if the excitation level is lower.

A closed loop control system has been developed to automatically tune the resonant frequency of this micro-generator to match the ambient vibration [17]. A new and more intelligent control system is currently being built to reduce the power consumption of the tuning mechanism. We aim to build a tunable vibration energy harvesting device with a completely self-powered tuning system having a useful net output power for wireless sensor nodes.

\section{References}

[1] M.R. Nezhad-Ahmadi, G. Weale, A. El-Agha, D. Griesdorf, G. Tumbush, A Hollinger, M. Matthey, H. Meiners, S. Asgaran, A 2 mW $400 \mathrm{MHz}$ RF transceiver SoC in $0.18 \mu \mathrm{m}$ CMOS technology for wireless medical applications, in: Radio Frequency Integrated Circuits Symposium, IEEE, 2008, pp. 285-288.

[2] A.C.W. Wong, G. Kathiresan, C.K.T. Chan, O. Eljamaly, O. Omeni, D. McDonagh, A.J. Burdett, C.C. Toumazou, A $1 \mathrm{~V}$ wireless transceiver for an ultra-low-power SoC for biotelemetry applications, IEEE Journal of Solid-State Circuits 43 (7) (2008) 1511-1521.

[3] B.C. Norman, Power options for wireless sensor networks, IEEE Aerospace and Electronic Systems Magazine 22 (4) (2007) 14-17.

[4] T. Huesgen, P. Woias, N. Kockmann, Design and fabrication of MEMS thermoelectric generators with high temperature efficiency, Sensors and Actuators A: Physical 145-146 (2008) 423-429.

[5] S.P. Beeby, M.J. Tudor, N.M. White, Energy harvesting vibration sources for microsystems applications, Measurement Science and Technology 17 (2006) 175-195.

[6] S. Roundy, P.K. Wright, J.J. Rabaey, A study of low level vibrations as a power source for wireless sensor nodes, Computer Communications 26 (2003) 1131-1144.

[7] C.B. Williams, R.B. Yates, Analysis of a micro-electric generator for microsystems, Sensors and Actuators A 52 (1996) 8-11.

[8] I. Sari, T. Balkan, H. Kulah, An electromagnetic micro power generator for wideband environmental vibrations, Sensors and Actuators A: Physical 145-146 (2008) 405-413.

[9] S.M. Shahruz, Design of mechanical band-pass filters for energy scavenging, Journal of Sound and Vibration 292 (2006) 987-998.

[10] V.R. Challa, M.G. Prasad, Y. Shi, F.T. Fisher, A vibration energy harvesting device with bidirectional resonance frequency tunability, Smart Materials and Structures 17 (2008) 015035.

[11] E.S. Leland, P.K. Wright, Resonance tuning of piezoelectric vibration energy scavenging generators using compressive axial preload, Smart Materials and Structures 15 (2006) 1413-1420.

[12] R.D. Blevins, Formulas for Natural Frequency and Mode Shape, Krieger Publishing Company, Malabar, FL, 2001. 
[13] D. Zhu, Methods of Frequency Tuning Vibration Based Micro-Generator, PhD thesis, University of Southampton.

[14] G. Akoun, J.P. Yonnet, 3D analytical calculation of the forces exerted between two cuboidal magnets, IEEE Transaction Magnetism 20 (5) (1984) 1962-1964.

[15] F.B. Hildebrand, Introduction to Numerical Analysis, Tata McGraw-Hill, Inc., 1979.

[16] S.P. Beeby, R.N. Torah, M.J. Tudor, P. Glynne-Jones, T. O’Donnell, C.R. Saha, S. Roy, A micro electromagnetic generator for vibration energy harvesting, Journal of Micromechanics and Microengineering 17 (2007) 1257-1265.

[17] D. Zhu, S. Roberts, M.J. Tudor, S.P. Beeby, Closed loop frequency tuning of a vibration-based micro-generator, in: Proceedings of PowerMEMS 2008+ microEMS2008, Sendai, Japan, November 9-12, 2008, pp. 229-232.

\section{Biographies}

D. Zhu obtained a BEng in Information and Control Engineering from Shanghai Jiao Tong University, China, in 2004. He was awarded his MSc and PhD in University of Southampton, UK in 2005 and 2009, respectively. He is currently a research fellow in Electronic Systems and Devices Group, School of Electronics and Computer Science, University of Southampton, UK.

S. Roberts obtained a PhD in optics in 1996 and held a technical management position in silicon integrated optics at Bookham Technology. He joined Perpetuum Ltd. as Technical Manager in 2005. Stephen is responsible for the development of the vibration energy-harvesting technology, managing the modelling and design of the range of micro-generators.

M.J. Tudor obtained a BSc (Eng) in electronic and electrical engineering from University College London and a PhD in physics from Surrey University. In 1987, he joined Schlumberger Industries working first at their Transducer Division in Farnborough and then their Research Centre in Paris, France. In 1990, he joined the University of Southampton as a lecturer and his research interests covered optical fibre sensors and micromachined sensors. In 1994, he moved to ERA Technology becoming the microsystems program manager. In 2001, he returned to the School of Electronics and Computer Science, University of Southampton to pursue university based research in microsystems. He is currently a principle research fellow. He has over 70 publications and is both a chartered physicist and engineer.

S.P. Beeby obtained his BEng (Hons) in mechanical engineering in 1992 and was awarded his PhD in 1998. He is currently a reader in the School of Electronics and Computer Science and is researching in the field of micro-electro-mechanical systems (MEMS). In particular, his research involves the development of fabrication processes whereby thick-film piezoelectric materials can be combined with micromachined silicon structures. His skills include the finite element modelling and design of MEMS devices, silicon processing and MEMS packaging, and testing. He currently has over 150 publications in the field. 